\title{
Designing and Additive Manufacturing A Prototype for A Novel Instrument for Mandible Fracture Reduction
}

\section{Risto Kontio ${ }^{2}$, Roy Björkstrand ${ }^{1}$, Mika Salmi ${ }^{1}$, Markku Paloheimo ${ }^{1}$, Kaija-Stiina Paloheimo ${ }^{1}$, Jukka Tuomi ${ }^{1}$, Antti A. Mäkitie ${ }^{1,3 *}$}

${ }^{1}$ Aalto University, School of Science, BIT Research Centre, Otaniementie 17, Espoo, Finland

${ }^{2}$ Departments of Maxillofacial Surgery - Head \& Neck Surgery, Helsinki University Central Hospital and University of Helsinki, Fl-00029 HUCH, Helsinki, Finland

${ }^{3}$ Otolaryngology - Head \& Neck Surgery, Helsinki University Central Hospital and University of Helsinki, Fl-00029 HUCH, Helsinki, Finland

\begin{abstract}
Background: Industrial additive manufacturing (AM) methods are currently used for various medical applications. AM makes possible the designing of custom made and even patient specific instruments. We aimed at planning and additive manufacturing a prototype of a novel instrument for mandible fracture correction.

Materials and Methods: The fracture reduction forceps were designed using the Parametric Computer Aided Design (CAD) system. The three dimensional (3D) data of the planned instrument were transferred to the AM machine using stereo lithography format. The final physical object was sintered using direct metal laser sintering.

Results: The reduction forceps were manufactured in three separate units: combination of the handle and the jaw, left and right handed versions, and the bar. The bar was a part of a hinge mechanism to fixate the units together. In the first prototype version the locking system design between the bar and the handles was functioning insufficiently although in the virtual CAD model it looked stable. Due to this handicap, we were not able to achieve experience from a clinical setting. We are currently in the process of re-designing the instrument prototype and manufacturing new versions. Thus, one of our findings was that when designing and manufacturing special instruments one needs to be prepared to produce several versions of the prototype, as the first physical model will likely not fulfill all the requirements set for the innovation.

Conclusions: A process was developed and tested where a novel surgical instrument that fulfilled the theoretical requirements of a certain specific facial trauma case was designed and manufactured. The advantage of CAD - AM process is that the instrument can be tested immediately and re-manufactured directly if necessary. Using these new technologies, it is currently possible to manufacture patient and operation specific instruments. This advantage opens new views not only for fracture surgery but also for other surgical fields.
\end{abstract}

Keywords: Direct metal laser sintering (DMLS); Additive manufacturing; Rapid prototyping; Surgery

\section{Introduction}

Additive manufacturing (AM) technologies were originally developed for industrial rapid prototyping purposes in the late 1980's. One of their key features is that manufacturing of a physical object is performed in a fully automated process based on a computerized threedimensional (3D) model. Furthermore, a layer-wise manufacturing strategy is applied, which makes it possible to create highly complicated parts in hours. [1]

Based on the many advantages of AM technologies, medical applications have now also become available. These vary from preoperative models [2], inert implants [3], supportive guides [4], to special tools and instruments.

Computed aided design (CAD) and planning is becoming more popular in modern surgery. Use of $3 \mathrm{D}$ modeling can aid in the visualization of the anatomy of the injured or deformed area. The vital structures and the deformity at the surgical site are identified in a 3D virtual model. The dimensions can be measured with great accuracy. Finally, using 3D CAD the actual operation and suitable surgical approach can be simulated, if needed, several times preoperatively. All these issues improve the quality control of the surgery. In addition to computerized planning, patient specific implants (PSI) can be designed and even manufactured directly using CAD and AM techniques. PSI implants are already available in otolaryngology, maxillofacial and neurosurgery. Materials that can be additive manufactured include for example titanium as an implant material and cobalt chrome for dental applications.

These innovations now make it possible to create new tools and instruments for patient specific surgical procedures and even new surgical methods. The main requirements for a good surgical instrument are that they are precise, easy to handle and convenient to use. An additional benefit is if they can function as a guidance device, i.e. if they can give control and guide the surgeon in a particular phase of surgery. The first two properties are widely available also when using stock instruments. However, the drawback of a stock instrument is that it is designed for average circumstances. In the cranial and maxillofacial region the $3 \mathrm{D}$ anatomy is of a great complexity and the surgery is often performed in a cavity or behind a vital anatomical structure resulting in a poor visibility and a limited approach, reach and mobility of the average stock instrument. Furthermore, a stock instrument can rarely act as a guidance device.

A patient and operation specific instrument (POSI) could give additional support to high quality surgery. The POSI could be designed and manufactured simultaneously with computerized planning and implant design. The focus for the design is that it fits the surgical site precisely, does not interfere with the surgical view, and facilitates and steers the process of surgery.

The aim of the present study was to design and additive manufacture a prototype of a novel instrument for mandible fracture correction. The starting point of this design was to create a forceps with the jaws perpendicular to the axial line of the instrument. The additional aims were to design the handles not in a straight line but slightly in oval

*Corresponding author: Antti A. Mäkitie, Department of Otolaryngology - Head \& Neck Surgery, Helsinki University Central Hospital, P.O.Box 220, Helsinki, Finland, E-mail: antti.makitie@helsinki.fi

Received January 02, 2012; Accepted January 30, 2012; Published February 05, 2012

Citation: Kontio R, Björkstrand R, Salmi M, Paloheimo M, Paloheimo KS, et al. (2012) Designing and Additive Manufacturing A Prototype for A Novel Instrument for Mandible Fracture Reduction. Surgery S1:002. doi:10.4172/2161-1076.S1-002

Copyright: ( 2012 Kontio R, et al. This is an open-access article distributed under the terms of the Creative Commons Attribution License, which permits unrestricted use, distribution, and reproduction in any medium, provided the original author and source are credited. 
shape axially and also, to design curved tips of the jaws in order to get better grip for fracture correction.

\section{Materials and Methods}

The design of the novel surgical instrument was created through a user-driven innovation process. The engineers and the surgeon first specified the difficulties in corrective mandibular operations where standard instruments are used to correct a mandibular fracture. Then new design and manufacturing possibilities - knowledge domain of the engineers - were presented and reflected against this specified surgical procedure. This co-operative work revealed the requirements for an optimal operation specific instrument.

Using CAD system two conceptual models were created to satisfy the requirements set for the novel instrument. The jawbone forceps was designed using Parametric Computer Aided Design (CAD) system Solid works 2009-2010 educational version (Dassault Systèmes, VélizyVillacoublay, France) (Figure 1). In collaboration with the surgeon the instrument model version for the prototype manufacturing process was then chosen. This decision was made on the available 3D geometric representation of the designed instrument (Figure 2).

After this designing phase, the 3D data of the planned instrument were transferred to the additive manufacturing machine using stereolithography (STL) format. STL describes the 3D surface geometry of an object using triangles and it is the most de facto standard commonly used interface for this purpose. Triangular facets are represented by X-, $\mathrm{Y}$ - and Z-coordinates of each vertex and there is also a normal vector for each single triangle. The normal vector indicates the side of the facet that is outside the object [5].

Additive manufacturing method was selected for the process because it is superior in manufacturing one-off parts. The forceps was sintered using direct metal laser sintering (DMLS). DMLS is an AM

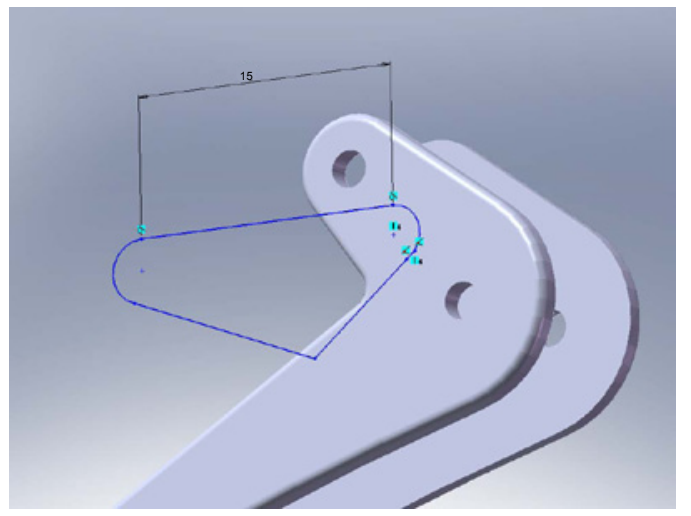

Figure 1: Representative illustration of design work using 3D CAD.

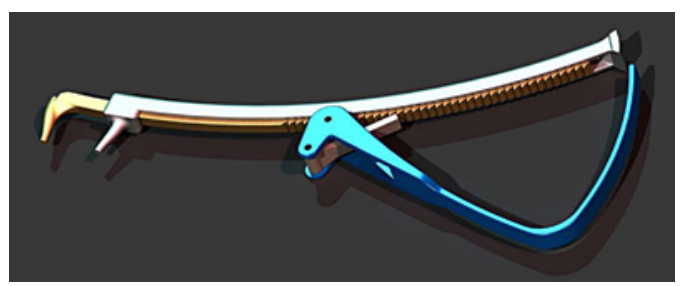

Figure 2: Visualization of the designed forceps for mandible fracture reduction. process where metal parts are manufactured directly from a 3D CAD model layer by layer.

During DMLS, a thin layer of powder material is first spread at the bottom plate of a build chamber. A high-powered laser is then focused with an in-build chamber using optics. Laser fuses the metal powder into a solid part. After the layer is finished a build platform lowers a layer thickness and a wiper blade is used to spread new powder layer over the build platform and the process then starts from beginning [6].

The machine used was Eosint M270 (Electro Optical Systems, Krailling, Germany) with a layer thickness of $0.2 \mathrm{~mm}$. The selected material was EOS Stainless Steel PH1 (Electro Optical Systems, Krailling, Germany) because of its high hardness and strength.

\section{Results}

The reduction forceps were manufactured in three separate units: combination of the handle and the jaw, left and right handed versions, and the bar. The bar was a part of the hinge mechanism that also connected the first two abovementioned units together. The instrument itself was then manually assembled (Figure 3 ). Due to rough surfaces of the units, the bar and the bar hole had to be manually polished to enable function of the hinge mechanism. The locking design between the bar and handles was insufficient although in the virtual CAD model it seemed stable enough. To lock rigidly the bar to the handles the bar should either be duplex (two parts) or should have screw threads. The bar did not compress the handles together strong enough but instead allowed lateral movement between structures. Because of this instability of the hinge, also the main locking mechanism was insecure. Due to this handicap, we were not able to achieve any clinical experience with this POSI reduction forceps.

\section{Discussion}

We describe the designing and AM process of a prototype of a novel surgical instrument to be used in mandible fracture correction. The multidisciplinary teamwork at various phases of this process represents the basis for innovative developmental work in the area of surgical instruments. These manufacturing methods allow production of both patient specific objects and surgeon specific instrumentation.

Modern mandible fracture surgery is performed almost exclusively intraorally and an extraoral approach is avoided because of the visible facial scars. This technique significantly limits the approach to the fracture site and new innovations regarding the surgical technique are warranted. One of the most difficult areas in this setting is the mandible angle where approximately $20 \%$ - $25 \%$ of mandible fractures are located. This site at the far back of the oral cavity is bordered by strong biting muscles i.e. pterygoid and masseter muscles. The golden principles for fracture surgery are accurate reduction of the fracture and stable maintenance until proper plate fixation of the fracture is performed. The reduction forceps available at the moment are designed for flat surfaces such as anterior mandible fractures, where the surgical area is near the orifice of the mouth. The commercial stock forceps are scissorlike i.e. the jaws are along the axial line of the instrument. During the reduction and maintenance phases, the handles of the instrument should be directed through the mouth orifice between the patient's lips. However, this type of design does not allow proper instrumentation in the posterior part of the oral cavity, since the forceps cannot be placed perpendicular to bone without penetrating the handles through facial skin. Currently, fractures of the mandible angle are repositioned either using the fixation plate as an implement to reduce the fracture or with reduction forceps introduced transcutaneously to the fracture site. 


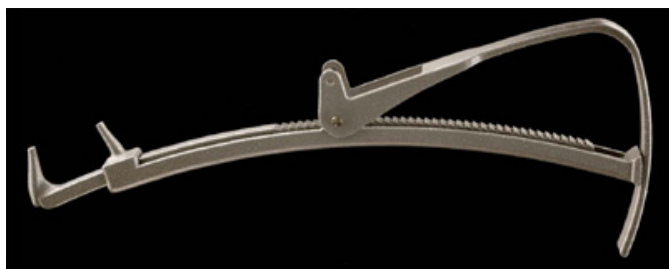

Figure 3: The additive manufactured forceps prototype.

The primary idea of the described prototype innovation process was that the features of the novel forceps would allow the instrument to be introduced through the mouth orifice while still enabling reduction of the fracture and holding this reduced fracture position until fixation with bone plates. The oval axial line design of the handles were planned to retain the buccal soft tissues and muscles in order to maintain good visibility throughout the surgical procedure. Finally, if the tips of the forceps would get a firm grip of the bone fragments this would allow active manipulation of the fracture with the forceps and thus make the reduction easier compared to the use of a stock instrument with weaker grip.

Additive manufacturing is an expensive technology when multiple parts are needed. On the other hand, custom made objects are unique and therefore patient and/or surgeon specific. Traditional manufacturing methods for personalized needs also cause high expenses due to needed manufacturing tooling etc. One possibility to overcome this aspect of high expenses would be to use standard instruments with interchangeable tips or handles, which are designed and produced with AM technology.

In the present study DMLS technology was tested to manufacture a prototype of an instrument and its related mechanics. It is well known that AM technologies do not result in as accurate work pieces as conventional numerically controlled machining methods.

However, our study demonstrated that the manufactured parts and the assembled instrument fulfilled the accuracy requirements related to this type of an object.

Single work pieces like e.g. drill guides are easier to design and manufacture with various AM technologies compared to multicomponent tools warranting assembly and properly functioning mechanics. Namely, a certain tolerance factor has to be added in the design of components with a mechanical function. The present study confirms this as the AM tolerances were taken into account when designing the novel functioning features of the tool.

Another important factor in the actual AM process planning is communication between the surgeon and the AM engineer. Orientation of various parts in the AM process influences their surface properties and the accuracy of various details of the object. Thus, it is essential that AM operator will be informed about the planned mechanics of an instrument and its final use. We have also found that when designing and manufacturing special instruments one needs to be prepared to produce several versions of the prototype, as the first physical model will likely not fulfill all the requirements set for the innovation. We and other groups have used computer aided modeling and simulation to analyze and optimize an instrument prototype in order to prove its feasible function, reliable performance, and mechanical advantage [7]. One option would be to use stereolithography prototyping to create partially functioning plastic models for subjective assessment of for example functional and ergonomic feasibility [8].

We conclude that we were able to design and manufacture an instrument that fulfilled the theoretical requirements of this specific case. Successful clinical experiences are needed to prove this prototype clinically applicable and useful. However, the present study showed that with careful multidisciplinary planning and design it is currently possible to manufacture POS instruments. This advantage opens new views not only for fracture surgery but also for other surgical fields.

\section{Acknowledgments}

The authors would like to thank the Finnish Funding Agency for Technology and Innovation (Tekes), Helsinki University Central Hospital Research Funds, DeskArtes, EOS Finland, Inion, Planmeca and LM-Instruments for financing the present research and M.Sc. (tech.) Pekka Paavola for the photographs. This study was part of the BIOMAN II research project, grant number 40079/07.

\section{References}

1. Levy GN, Schindel R, Kruth JP (2003) Rapid Manufacturing and Rapid Tooling with Layer Manufacturing (LM) Technologies. State of The Art and Future Perspectives. Annals of the CIRP 52: 589-609.

2. McDonald JA, Ryall CJ, Wimpenny DI (2001) Rapid Prototyping Casebook Professional Engineering Publishing Limited, London, ISBN 1860580769.

3. Janssens M, Poukens J (2007) Rapid technologies in medicine: What can, can't be done and why. Proceedings of the International Conference on Competitive Manufacturing, Stellenbosch, South Africa.

4. Ng P, Lee PSV, Goh JCH (2002) Prosthetic sockets fabrication using rapid prototyping technology. Rapid Prototyping Journal 8: 53-59.

5. Chua CK, Gan JGK, Tong M (1997) Interface between CAD and rapid prototyping systems. A study of existing interfaces. International Journal of Advanced Manufacturing Technology 13: 566-570.

6. Khaing MW, Fuh JYH, Lu L (2001) Direct metal laser sintering for rapid tooling processing and characterisation of EOS parts. Journal of Materials Processing Technology 113: 269-272.

7. Li JZ (2009) Computational modeling and design of a new open clip surgical instrument. Int J Surg 7: 373-376.

8. Gonzalez-Cota A, Kruger GH, Raghavan P, Reynolds PI (2010) Computationa modeling and prototyping of a pediatric airway management instrument. Anesth Analg 111: 649-52. 